\title{
Design and Measurement of Microwave Absorbers Comprising Resistive Frequency Selective Surfaces
}

\author{
Meng Zhang, Tian Jiang, Yijun Feng* \\ School of Electronic Science and Engineering, Nanjing University, Nanjing, China \\ Email: viffeng@nju.edu.cn
}

Received 16 April 2014; revised 12 May 2014; accepted 7 June 2014

Copyright (C) 2014 by authors and Scientific Research Publishing Inc.

This work is licensed under the Creative Commons Attribution International License (CC BY).

http://creativecommons.org/licenses/by/4.0/

c) (i) Open Access

\section{Abstract}

Frequency selective surfaces (FSSs) have been successfully used in constructing microwave absorbers which demonstrate the ability of modifying and improving its absorbing performances. In this paper, microwave absorber based on ferromagnetic nano-films has been proposed and investigated with a structure similar to that of the Salisbury screen except that the resistive sheet is replaced by FSS of square patch array of the nano-film. We have explored the FSS absorber from three aspects: equivalent circuit model, electromagnetic full-wave simulation and the actual sample measurement. By carefully tuning the patch size, the thickness of the dielectric spacer and the specification of the ferromagnetic nano-film, we obtain the optimized performance of broadband microwave absorbing. Due to the light weight of the nano-films, the proposed absorbers can achieve an ultra-low surface density less than $0.25 \mathrm{~kg} / \mathrm{m}^{2}$.

\section{Keywords}

Salisbury Screen, Frequency Selective Surface, Equivalent Circuit Model, Electron Beam Evaporation

\section{Introduction}

The Salisbury screen is a passive microwave absorber which is constructed by placing a single thin resistive sheet a distance of quarter wavelength above a perfect electrically conducting (PEC) ground plane [1]. At resonance the impedance of the conducting plate is transformed to an open circuit at the resistive sheet which is normally $377 \Omega /$ square. The incident microwave has a $377 \Omega$ impedance in free space and is therefore totally

\footnotetext{
*Corresponding author.
} 
absorbed by the loading sheet [2] [3]. The PEC ground plane can be replaced by a high-impedance surface (HIS), then a phase delay greater than 180 degrees can be obtained and the physical thickness can be reduced [4].

The absorbing bandwidth of the Salisbury screen can be increased by employing more resistive sheets spaced approximately quarter wavelength apart, often referred to as a Jaumann absorber [5], or by placing dielectric slabs above and below the resistive sheets [2]. These structures are, however, unsuitable for deployment in applications where the major design driver is to reduce physical thickness. Recently, it was shown that an analysis of the absorbing structure by a simple equivalent circuit that allowed a detailed explanation of the key parameters for the design of the absorber. The authors quantified the relationship between the surface resistance of the ink film composing the screen and the reflectivity bandwidth [6].

The work described in this paper was motivated by the need to widen the absorption band of the Salisbury screen absorber. The resistive Salisbury screen was replaced by a high-impedance surfaces (HIS) comprising lossy frequency selective surfaces (FSS) such as a periodic array of conductive patch elements. The structure under analysis was described by means of an equivalent circuit approach. Commercial full wave electromagnetic (EM) wave simulation based on finite integration method [7] was employed to optimize the performance of the FSS and to obtain the physical dimensions of the unit cells for an absorber design. The computed results were shown to be in agreement with free-space measurements of the EM wave reflectivity.

\section{Thin Film Salisbury Screen Absorber}

The Salisbury screen was invented by American engineer Winfield Salisbury in 1952 [8] [9]. A Salisbury screen consists of three layers: a ground plane which is the metallic surface that needs to be concealed, a lossless dielectric of a precise thickness (a quarter of the wavelength of the radar wave to be absorbed), and a thin lossy screen. At resonance, the short circuit impedance of the ground plane is transformed to an open circuit at the position of the lossy screen whose surface impedance is normally $377 \Omega$ /square. Therefore, the structure presents a perfect impedance match to free space at one frequency and strongly absorbs the incident plane waves.

In this paper, the first attempt is to establish a Salisbury screen with a resistive sheet composed of alternating $\mathrm{Fe} / \mathrm{SiO}_{2}$ nano-films prepared with electronic-beam evaporation. The absorber consists of the Salisbury screen over a thin grounded dielectric slab (see Figure 1(a)).

By using the high energy electron beam evaporation, the $\mathrm{Fe}$ and $\mathrm{SiO}_{2}$ layers were alternating deposited on the polyimide film with thickness of about $40 \mu \mathrm{m}$, forming a multilayer films. As shown in Figure 1(b), the scanning electron microscope (SEM) picture of the cross section clearly demostrates the structure with alternating deposition of $\mathrm{Fe}$ or $\mathrm{SiO}_{2}$ film. The effective sheet impedance of the multilayer film can be controlled by the number of layers and the thickness of the $\mathrm{Fe}$ and $\mathrm{SiO}_{2}$ layers.

The use of substrate with high dielectric permittivity could reduce the thickness $d$ of the absorber. At resonance frequency according to [10], the propagation constant can be computed analytically as follows:

$$
\beta=2 \pi \frac{\sqrt{\mu_{r} \varepsilon_{r}}}{\lambda} .
$$

After some analytical manipulations, the relationship between the permittivity and the thickness $d$ of the dielectric layer can be derived. In Figure 2 the reflectivity of the Salisbury screen obtained by full wave simulation and by free-space measurement is reported. The results are not the optimal ones with some deviation with each other because the ferromagnetism has some influence on the impedance of the Salisbury screen. But they are presented without significant differences in order to verify the design methodology.

\section{Microwave Absorber with Resistive Frequency Selective Surface}

There are significant disadvantages to the concept which have limited the use of Salisbury screens. One is that the above simple Salisbury screen design works only at a single radar frequency or for narrow frequency band. More complicated multilayer Salisbury designs can cover a much broader band of frequencies, but only by increasing the thickness, and the best covers only a fraction of the radar spectrum. Another problem is the thickness of the screen itself. Radar wavelengths usually range between $10 \mathrm{~cm}$ and $1 \mathrm{~mm}$, thus the thickness of the screen (a quarter-wavelength) must be at most $2.5 \mathrm{~cm}$, and multilayer screens are much thicker. So here our second attempt is to use a high-impedance surfaces (HIS) comprising lossy frequency selective surfaces (FSS) with a periodic array of conductive patch elements to replace the resistive Salisbury screen. 


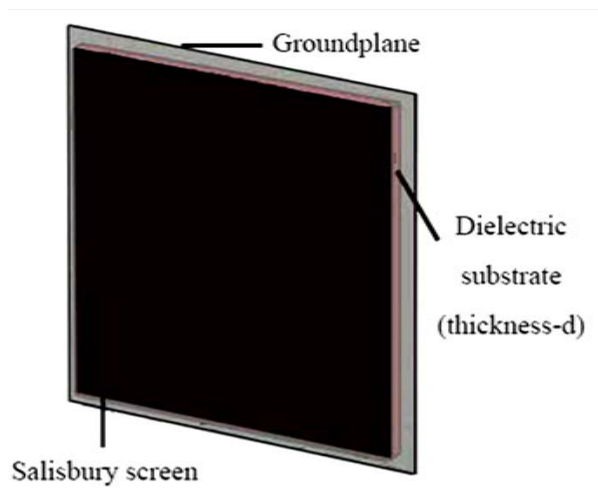

(a)

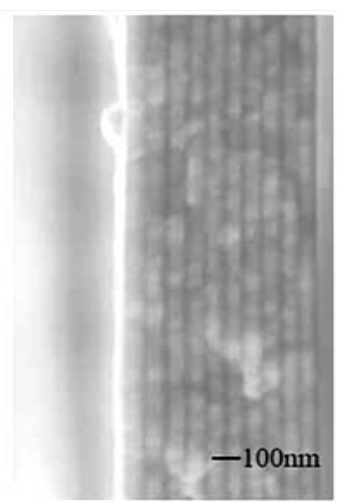

(b)

Figure 1. Three-dimensional sketch of the Salisbury screen (a) and SEM cross section drawn (b) of the multilayer thin films (Fe: dark grey area, $\mathrm{SiO}_{2}$ : grey area).

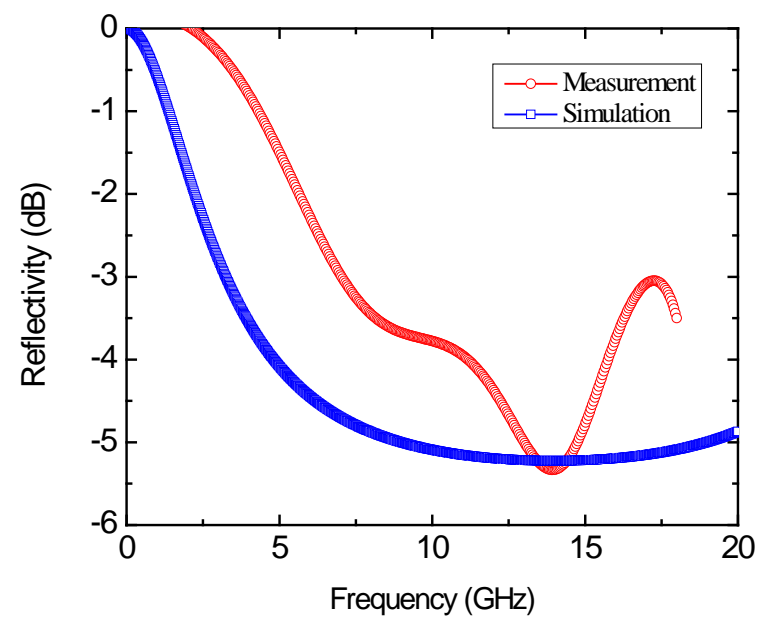

Figure 2. Measured reflectivity of the sample shown in Figure 1 compared to the simulated result.

The proposed absorber consists of a conventional high impedance surface comprising lossy frequency selective surfaces over a thin grounded dielectric slab (see Figure 3(a)). The equivalent circuit model of the FSS absorber is plotted in Figure 3(b). According to [6], the FSS array made up of capacitive cell of square patch, behaves as a capacitor in the low frequency region but its impedance becomes inductive after the first resonance. The impedance of a lossy FSS can be represented through a series RLC circuit as

$$
Z_{\mathrm{FSS}}=R-j\left(\frac{1-\omega^{2} L C}{\omega C}\right) .
$$

The shape of FSS influences the values of $L$ and $C$ parameters. The surface impedance of the absorbing structure $Z_{R}$ is equal to the parallel connection between the FSS impedance $Z_{\mathrm{FSs}}$ and the surface impedance of the grounded dielectric slab $Z_{d}$ :

$$
Z_{R}=\frac{Z_{d} Z_{\mathrm{FSS}}}{Z_{d}+Z_{\mathrm{FSS}}} .
$$

The thin grounded dielectric slab behaves as an inductance when the dielectric thickness is much less than a quarter wavelength. Its impedance can be computed analytically as

$$
Z_{d}=j Z_{m}^{\mathrm{TE}, \mathrm{TM}} \tan (\beta d),
$$




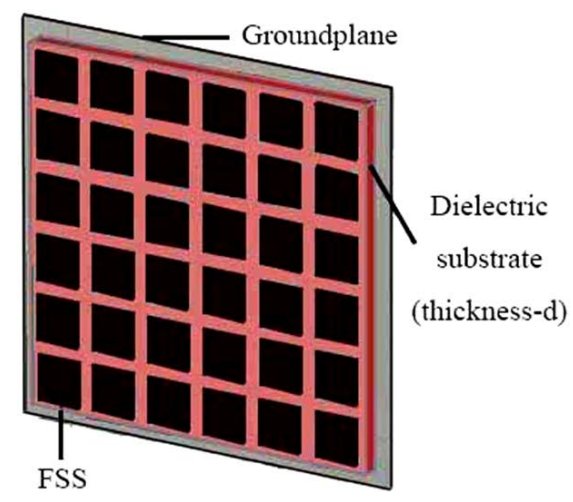

(a)

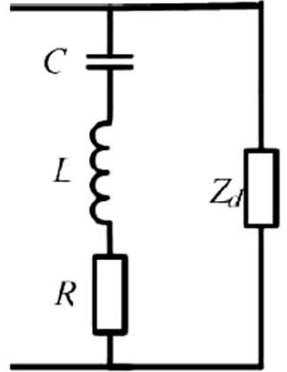

(b)

Figure 3. Three-dimensional sketch (a) and its equivalent circuit (b) of the proposed FSS absorber.

where $Z_{m}^{\mathrm{TE}}=\left(\omega \mu_{r} \mu_{0}\right) / \beta, Z_{m}^{\mathrm{TM}}=\beta /\left(\omega \varepsilon_{r} \varepsilon_{0}\right)$ are the characteristic impedances of the slab for TE and TM polarization, respectively. $\beta=\sqrt{k^{2}-k_{t}^{2}}$, which is the propagation constant along the normal direction of the slab, and $k_{t}=k_{0} \sin (\theta)$ is the transverse wave number with $\theta$ representing the incidence angle of the incoming wave with respect to the surface normal.

After some analytical manipulations, the real and the imaginary part of the input impedance can be derived:

$$
\begin{gathered}
\operatorname{Re}\left\{Z_{R}\right\}=\frac{R Z_{d}^{2}}{\left[\frac{1-\omega^{2} L C}{\omega C}-Z_{d}\right]^{2}+R^{2}}, \\
\operatorname{Im}\left\{Z_{R}\right\}=\frac{\left[-\omega L Z_{d}\right] \cdot\left[\frac{1-\omega^{2} L C}{\omega C}-Z_{d}\right]+\left[\frac{1}{\omega C} \cdot Z_{d}\right] \cdot\left[\frac{1-\omega^{2} L C}{\omega C}-Z_{d}\right]+R^{2} Z_{d}}{\left[\frac{1-\omega^{2} L C}{\omega C}-Z_{d}\right]^{2}+R^{2}} .
\end{gathered}
$$

When the inductive impedance of the substrate $Z_{d}$ and the imaginary part of the FSS impedance $\left(1-\omega^{2} L C\right) / \omega C$ assume the same value, the parallel circuit resonates and the impedance $Z_{R}$ becomes purely real (for thin substrates) and equal to:

$$
\operatorname{Re}\left\{Z_{R}\right\}=\frac{\left(Z_{m}^{\mathrm{TE}, \mathrm{TM}}\right)^{2} \tan ^{2}\left(k_{m}^{\mathrm{TE}, \mathrm{TM}} d\right)}{R} .
$$

In order to obtain absorption of the incoming waves, the impedance expressed in (7) should match the free space impedance $\zeta_{0}$, therefore we have

$$
R_{\mathrm{opt}}=\frac{\left(Z_{m}^{\mathrm{TE}, \mathrm{TM}}\right)^{2} \tan ^{2}\left(k_{m}^{\mathrm{TE}, \mathrm{TM}} d\right)}{\zeta_{0}^{\mathrm{TE}, \mathrm{TM}}} .
$$

The relation in (8) highlights the dependence of the optimal FSS resistance on the thickness and permittivity of the substrate. Through the circuital approach, in correspondence of the resonance frequency generated by the parallel between the capacitive impedance of the FSS and the inductive impedance of the grounded dielectric slab, it is evident that by increasing the substrate thickness, the value of the real part of the input impedance becomes larger. This allows synthesizing a good absorber in correspondence of the HIS resonances. As is well known, a grounded dielectric substrate behaves as a capacitance when its thickness becomes thicker than a quarter wavelength. It is therefore necessary employing a slightly thicker substrate to obtain a capacitive behavior in the frequency range of interest. In addition, at the center of the operating band, the substrate thickness equals quarter 
wavelength acting as a high-impedance wall while the FSS impedance turns from capacitive to inductive showing a purely real impedance. These circumstances allow the structure working as a conventional Salisbury screen [9] [10] at the center of the operating band [8].

To construct a proto-type, the resistive patch array was directly manufactured by electron beam evaporation of $\mathrm{Fe} / \mathrm{SiO}_{2}$ multilayer film with certain mask. It represents the lossy frequency selective surfaces with capacitive impedance. The FSS composed of the patch array is then loaded on a foamy grounded dielectric substrate (see the three-dimensional sketch of the proposed absorbing structure illustrated in Figure 3(a)).

A picture of the fabricated sample is shown in Figure 4. In this example, the FSS consists of a patch array with a periodicity of $21 \mathrm{~mm}$ and the ratio $P$ between the side length $L$ of the element and the cell periodicity $D$ equal to $18 / 21$. The surface resistance of the square patches is equal to $120 \Omega$ /square and the substrate is the commercial foam with permittivity equal to $\varepsilon_{r}=1.15$. The overall thickness of the absorber is $5 \mathrm{~mm}$. The value of lumped parameters ( $L, C$ and etc.) can be obtained by computing the equivalent circuit, and the lumped resistance can be derived after a full wave simulation of a freestanding FSS by computing the real part of the FSS impedance [11]. The bandwidth is comparable to the one achievable by a conventional Salisbury configuration. The measured reflectivity, compared with the simulated result, is plotted in Figure 5.

The designed absorber can achieve $-10 \mathrm{~dB}$ absorbing bandwidth up to $11 \mathrm{GHz}$ in the 2 - $18 \mathrm{GHz}$ frequency band. Obviously the result of the free-space measurement is even better than the result obtained by full wave simulation. So the influence on the impedance of the absorber induced from the ferromagnetism of the film is not significant. However, this represents a practical issue that need to be further investigated.

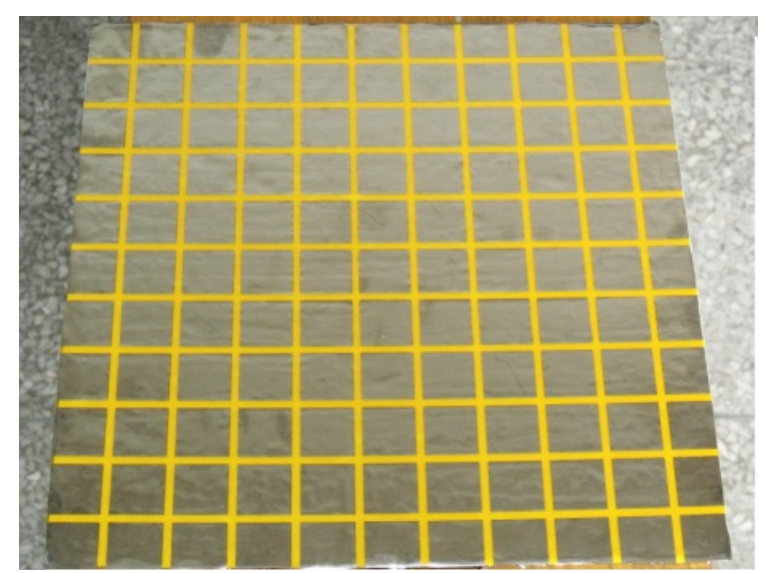

Figure 4. A sample of the absorber comprising lossy frequency selective surfaces.

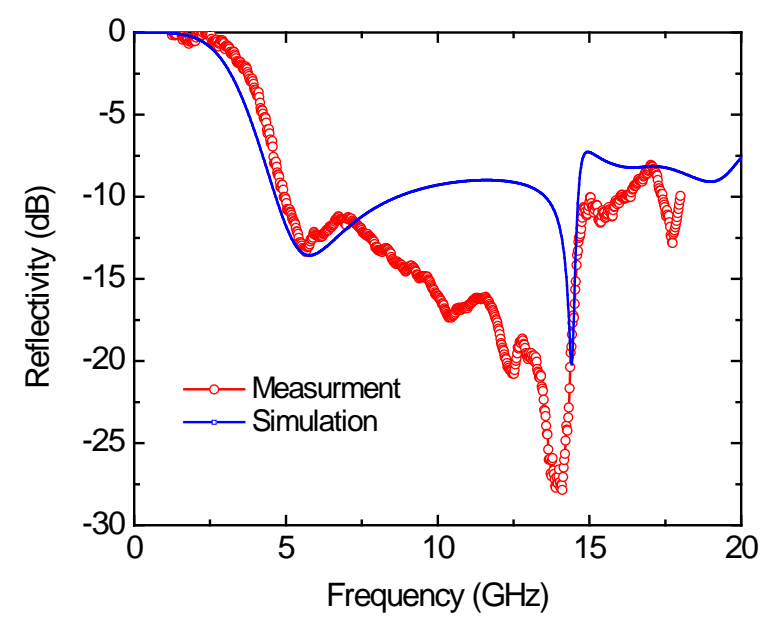

Figure 5. Measured reflectivity of the sample shown in Figure 3 compared to the simulated result. 


\section{Conclusions}

In this paper we have provided a detailed explanation of thin absorbers based on high-impedance surfaces. Different from the conventional Salisbury screen, the absorber consists of a lossy frequency selective surface placed above a grounded dielectric slab. The absorbing structure can be employed for synthesizing wideband absorbers that outperform the conventional Salisbury and Jaumann configurations. In the present work, we firstly show a resistive Salisbury screen that has been synthesized by an alternating $\mathrm{Fe} / \mathrm{SiO}_{2}$ nano-film prepared with electron beam evaporation. The reflection coefficient of the Salisbury screen obtained by full wave numerical simulation and by free-space EM wave measurement is reported and compared.

Secondly, a lossy FSS has been synthesized by manufacturing a resistive patch array through electron beam evaporation. The use of resistive patch provides a much simpler and lightweight structure with respect to other design including a large number of lumped resistors. The proposed absorber can achieve an ultra-low surface density less than $0.25 \mathrm{~kg} / \mathrm{m}^{2}$. By means of a lumped equivalent circuit, we have determined simple rules for choosing the optimal surface resistance of the FSS. Indeed, the surface resistance represents the key parameter of the structure. The working principle of wideband absorbers has been addressed by the model. Simulation and experimental results have been presented which have verified the analyzed configurations. The proposed design could find many applications in EMC and EMI technology.

\section{Acknowledgements}

This work is partially supported by the National Nature Science Foundation of China (61301017, 61371034, 61101011), the Key Grant Project of Ministry of Education of China (313029), and the Ph.D. Programs Foundation of Ministry of Education of China (20100091110036, 20120091110032), and partially supported by Jiangsu Key Laboratory of Advanced Techniques for Manipulating Electromagnetic Waves.

\section{References}

[1] Knott, E.F., Shaeffer, J. and Tuley, M. (2004) Radar Cross Section. SciTech Publishing Inc., Raleigh.

[2] Munk, B.A., Munk, P. and Pryor, J. (2007) On Designing Jaumann and Circuit Analog Absorbers (CA Absorbers) for Oblique Angle of Incidence. IEEE Transactions on Antennas and Propagation, 55, 186-193. http://dx.doi.org/10.1109/TAP.2006.888395

[3] Chambers, B. (1994) Optimum Design of a Salisbury Screen Radar Absorber. Electronics Letters, 30, $1353-1354$. http://dx.doi.org/10.1049/el:19940896

[4] Seman, F.C., Cahill, R. and Fusco, V. (2009) Low Profile Salisbury Screen Radar Absorber with High Impedance Ground Plane. Electronics Letters, 45, 10-12. http://dx.doi.org/10.1049/el:20093098

[5] Chambers, B. and Tennant, A. (1994) Design of Wideband Jaumann Radar Absorbers with Optimum Oblique Incidence Performance. Electronics Letters, 30, 1530-1532. http://dx.doi.org/10.1049/el:19941023

[6] Costa, F., Monorchio, A. and Manara, G. (2010) Analysis and Design of Ultra Thin Electromagnetic Absorbers Comprising Resistively Loaded High Impedance Surfaces. IEEE Transactions on Antennas and Propagation, 58, 15511558. http://dx.doi.org/10.1109/TAP.2010.2044329

[7] (2010) CST Microwave Studio. http://www.cst.com

[8] Munk, B.A. (2005) Frequency Selective Surfaces: Theory and Design. John Wiley \& Sons Ltd., Chichester.

[9] Salisbury, W.W. (1952) Absorbent Body for Electromagnetic Waves. US Patent No. 2599944 A.

[10] Fante, R.L. and Mccormack, M.T. (1988) Reflection Properties of the Salisbury Screen. IEEE Transactions on Antennas and Propagation, 36, 1443-1454. http://dx.doi.org/10.1109/8.8632

[11] Costa, F., Monorchio, A. and Manara, G. (2009) An Equivalent Circuit Model of Frequency Selective Surfaces Embedded within Dielectric Layers. Proceedings of the 2009 IEEE Antennas and Propagation Society International Symposium, Charleston, 1-5 June 2009, 1-4. 
Scientific Research Publishing (SCIRP) is one of the largest Open Access journal publishers. It is currently publishing more than 200 open access, online, peer-reviewed journals covering a wide range of academic disciplines. SCIRP serves the worldwide academic communities and contributes to the progress and application of science with its publication.

Other selected journals from SCIRP are listed as below. Submit your manuscript to us via either submit@scirp.org or Online Submission Portal.
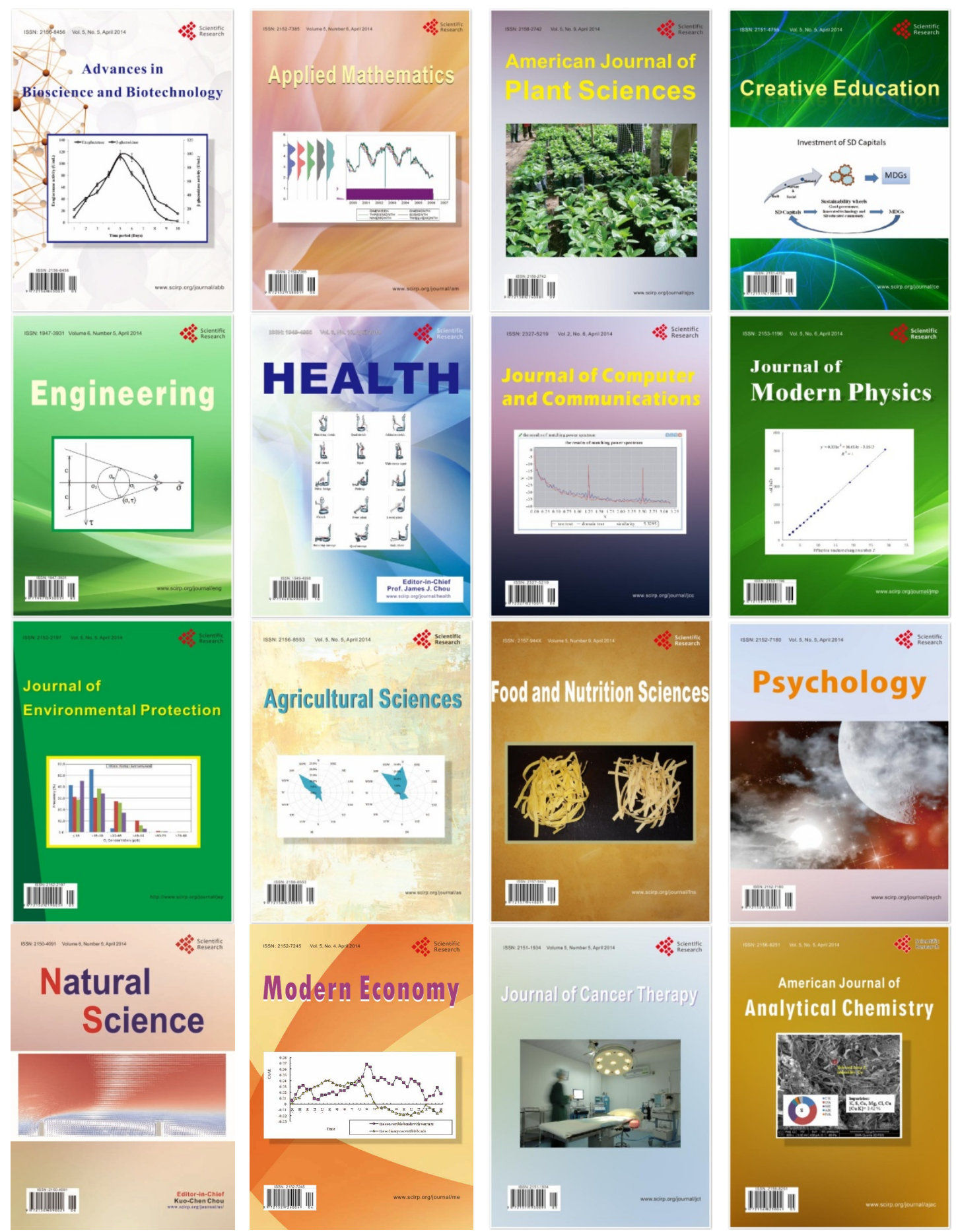